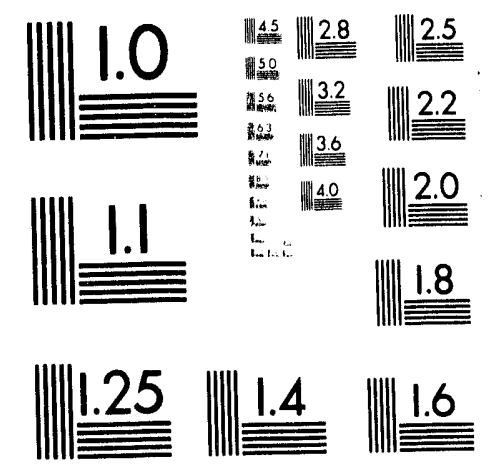



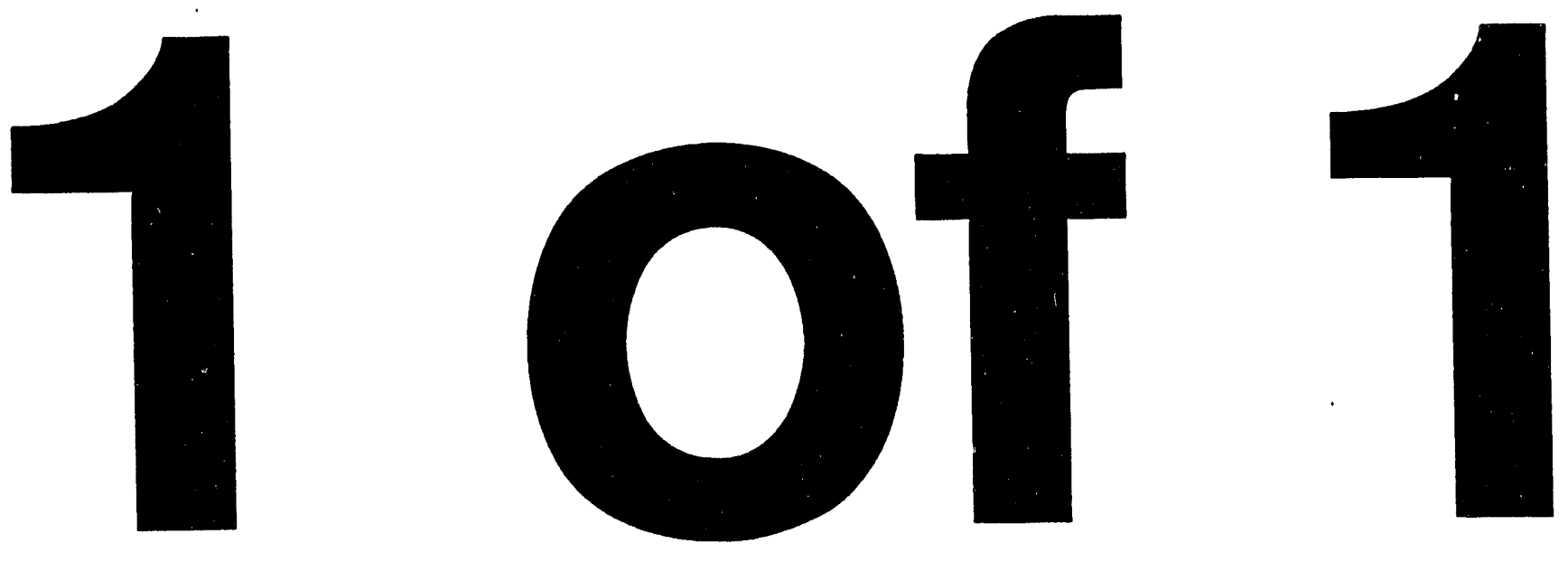


\title{
ENHANCED COHERENCE X-RAY LASER EXPERIMENTS AND SIMULATIONS
}

\author{
Alan S. Wan, Stephen B. Libby, Juan C. Moreno,
}

This paper was prepared for submittal to the 11th International Conference on Laser Interaction and Related Plasma Phenomena Monterey, CA

October 25-29, 1993

October 1993

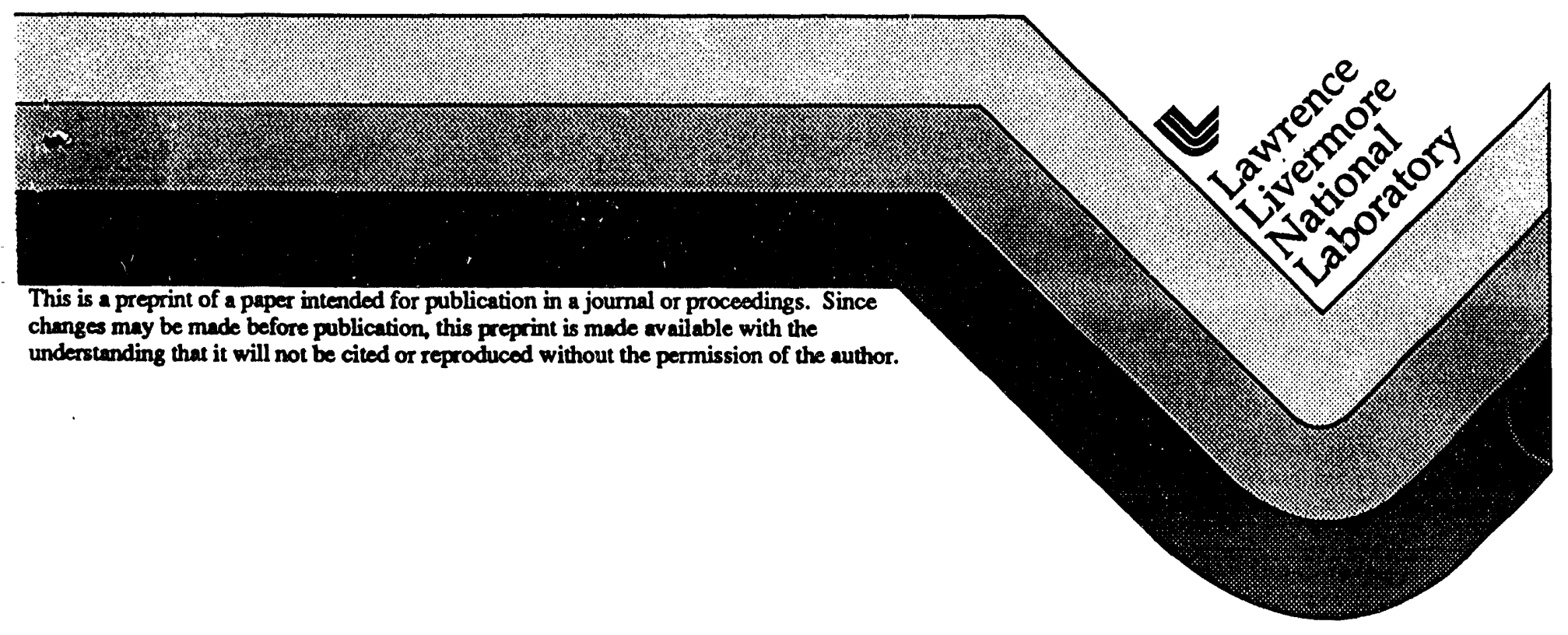

This is a preprint of a paper intended for publication in a journal or procedings. Since changes may be made before publication, this preprint is made available with the understanding that it will not be cited or reproduced without the permission of the author. 


\section{DISCLAIMER}

This document was prepared as an account of work sponsored by an agency of the United States Government. Neither the United States Government nor the University of California nor any of their employees, makes any warranty, express or implied, or assumes any legal liability or responsibility for the eccuracy, completeness, or usefulness of any information, apparajus, produch, or process disclosed. or represents that its use would not infringe privately owned rights. Reference herein to any specific commercial products, process, or service by trade name, trademark, manufacturer, or otherwise, does not necessarily constitute or imply its endorsement, recommendation, or favoring by the United States Government or the University of Califomia. The views and opinions of authors expressed herein do not necessarily state or reflect those of the United States Government or the University of Califomia, and shall not be used for advertising or product endorsement purposes. 


\title{
ENHANCED COHERENCE X-RAY LASER EXPERIMENTS AND SIMULATIONS*
}

\author{
Alan S. Wan, Stephen B. Libby, Juan C. Moreno \\ Lawrence Livermore National Laboratory \\ P. O. Box 808, Livermore, CA 94550
}

\begin{abstract}
Bright, spatially coherent x-ray lasers (XRLs) have applications in areas such as holography, interferometric imaging, and non-linear optics. Nominally, we can improve XRL coherence by either increasing the length or by reducing the aperture. Length can be increased by coupling multiple stages of XRLs or by using multilayer optics, but the effective gain length of an XRL is limited by refractive propagation and multilayer damages. The laser aperture is typically limited by the best-focused configuration defined by the driving optical lasers. Design of XRLs produced in exploding foil or slab configurations is further complicated by large spatial gain and $n_{e}$ inhomogeneities. This paper explores new XRL design that uses the concept of adaptive spatial filtering by geometric shaping to improve the transverse coherence. One example of such shaped XRLs is a bowtie. We present here computational and experimental characterization of shaped XRLs during laser plasma expansion by studying their hydrodynamic behaviors, ionization histories, and laser output intensities.
\end{abstract}

\section{INTRODUCTION}

XRLs $(1,2)$ have been considered for applications in the fields of microscopy, material processing, and as probing tools for plasma, atomic, and molecular physics phenomena. XRL applications in areas such as holography and non-linear optics require the laser to be both bright and spatially coherent. Although saturated XRLs have been demonstrated, $(3,4)$ XRLs are spatially incoherent with Fresnel numbers $~ 50 .(5)$ Improved coherence has been demonstrated by using multilayer mirrors and oscillator-amplifier configurations, $(4,6)$ but these multi-staged configurations are limited by uncertainties in refractive ray propagation and by multilayer damage threshold.(7) The recent proposal of using adaptive spatial filtering by geometric shaping( $(8)$ is an alternative technique that can produce saturated, single-staged, and diffraction-limited XRLs. These shaped lasers can be either stand-alone lasers or they can be used as oscillators driving larger aperture amplifiers in optical architectures.

This paper presents our recent theoretical, numerical, and experimental characterization of enhanced coherence XRLs. The experiments were carried out at the Nova 2-Beam Facility at Lawrence Livermore National Laboratory (LLNL) where we are characterizing the hydrodynamics, laser, and ionization balances of various shaped XRL configurations. In section 2 of this paper we present theoretical analysis of adaptive spatially filtered XRLs. In section 3 we present computational simulations of shaped XRLs, including laser-plasma interactions, hydrodynamics, atomic kinetics, and gain calculations. Section 4

* Work performed under the auspices of the U. S. DOE by LLNL under contract number W-7405ENG-48 and is partially supported by the Institute Sponsored Research Program. 
shows our experimental setup and presents the experimental results. We will summarize our findings and outline our future research directions in section 5 .

\section{THEORETICAL MODELING OF ADAPTIVELY SPATIALLY FILTERED XRLS}

In a typical slab XRL configuration, a best-line-focused optical laser (120 $\mu \mathrm{m}$ FWHM for Nova) illuminating a uniformly coated solid slab or foil target. The laser-driven plasma expands in two dimensions, with electron density $\left(n_{e}\right)$ gradients both parallel and perpendicular to the target surface. Laser photons propagate in a highly refractive medium and produce typical two-dimensional (2-D) laser divergences $\sim 10$ mrads in the blow-off direction and $\sim 20 \mathrm{mrad}$ in the transverse direction. ${ }^{(5)}$

Instead of letting the driving laser optics define the XRL aperture, the concept of adaptive spatial filtering of XRLs uses geometric shaping to control the laser aperture, such as the bowtie-shaped XRL shown in Fig. 1 with a bowtie (light region) deposited on a substrate made of a similar- $Z$ material (dark region). The neck of the bowtie acts as a narrow spatial filter where the high-gain rays must pass through. In the unsaturated regime, rays that traverse the longest gain regions have the possibility of attaining the greatest gain-lengths (GL) and highest output intensities. The neck of the bowtie laser acts as a narrow spatial filter where the high-GL rays must pass through. These high-GL rays have strong correlation between their angle of tilt and their transverse positions. Rays that travel outside of the bowtie neck achieve lower GLs and are also attenuated by surrounding materials. In a nonrefractive and uniform gain medium, the output of an unsaturated bowtie XRL would be weaker than that of a constant-width stripe XRL by the ratio of the neck area to the end area.

In the saturated regime, bowtie XRLs actually increase the coherent power by causing significant power extraction into only a few modes and by eliminating parasitic modes. In saturation the effective (or loaded) gain depends on the overall intensity pattern in a nonlinear and self-consistent way. The maximum gain of a saturated bowtie XRL is located at the neck region where the adaptive spatial filtering occurs. This effective spatial gain distribution maintains the correlation between ray tilt and transverse position at the laser output end that is needed to achieve high coherent power. Thus the energy content of a saturated XRL preferentially flows through the few Fresnel zones defined by the neck of the bowtie. In a non-refractive and uniform-gain medium, with all the energy extracted, the output intensity of a bowtie XRL would be weaker than that of a stripe XRL by the ratio of active gain volumes between the two geometries. However, the coherent power of a bowtie XRL would be much stronger than that of a stripe XRL.

A key issue in the performance of bowtie XRLs is our ability to maintain the bowtie shape during the plasma expansion. We have several design options for a more hydrodynamically stable shaped XRL. One way to fabricate a bowtie XRL is to deposit a bowtie-shaped thin film coating on a substrate made of similar- $Z$ material. Incoming optical laser overfills the bowtie XRL and producing a one-dimensional (l-D) blow-off near the XRL plasma. The substrate plasma provides a hydrodynamic tamper to the XRL plasma and maintains the bowtie shape during the lasing period.

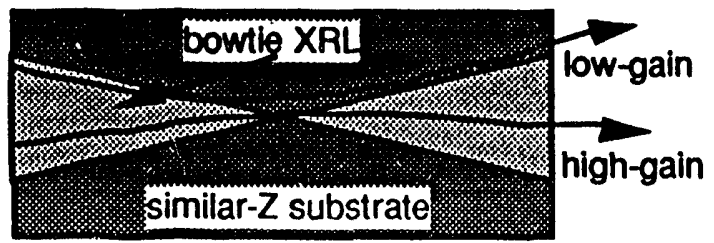

Figure 1. Bowtie XRL deposited on similar-Z solid substrate. High-gain rays pass through the bowtie neck and dominate the output. 


\section{NUMERICAL SIMULATIONS}

The modeling of XRL plasmas is periormed in stages. For collisionally dominated plasmas we can decouple the detailed level populations and line transfer physics from the rest of the problem. ${ }^{(9)}$ We typically use LASNEX ${ }^{(10)}$ to carry out the laser-deposition and hydrodynamics simulations. From LASNEX we obtain plasma characteristics, such as $T_{\mathfrak{e}}$ and $n_{\mathfrak{c}}$, and variables such as mesh positions and velocities. Using atomic kinetic post-processing codes such as GLF, $(9,11)$ we calculate the level populations and line transfer.

In our LASNEX simulation the incident laser (from the z-direction) is defined by a super-Gaussian spatial profile with FWHM set at the experimentally defined line width. A symmetry plane $(r=0)$ defines the center of the line focus geometry. The pulse shape matches the experimentally defined 600 ps square pulse with $20 \mathrm{TW} / \mathrm{cm}^{2}$ driving laser intensity at twice the Nd-glass frequency $($ or $0.53 \mu \mathrm{m}$ ), herein denoted as $2 \omega$. Figs. 2 and 3 compare the 2-D plasma expansions, at 500 ps into the laser pulse, between the slab XRL ( $120 \mu \mathrm{m}$ best-focused) and the stripe XRL (240 $\mu \mathrm{m}$ defocused overfilling an $120-\mu \mathrm{m}$ stripe) configurations. The dark lines mark the $60 \mu \mathrm{m}$ position at the target surfaces that correspond to the edge of the Ge stripe XRL plasma for Fig. 3 and the FWHM position of the bestfocused geometry of Fig. 2. The slab XRL plasma has large transverse-direction (parallel to target) velocities which result in larger transverse $n_{e}$ gradients. By overfilling the stripe, the Ge stripe XRL plasma is hydrodynamically tamped by the $\mathrm{Cu}$ plasma and the XRL plasma expansion is more 1-D. This 1-D expansion allows us to maintain the geometrically shaped XRL configuration we need for enhanced coherence. With better transverse confinement, the stripe plasma is both hotter and denser, with flatter $n_{e}$ profiles in both $r$ - and z-directions.

Most of the postprocessing are taken along a 1-D slice of the LASNEX mesh. From the LASNEX calculations we extract $T_{e}, T_{i}$, material density, mesh position and velocity, and the velocity gradient and use GLF and obtain the level populations and the small signal gains. The atomic processes include electron-ion and ion-ion collisions, photo-ionization and photo-

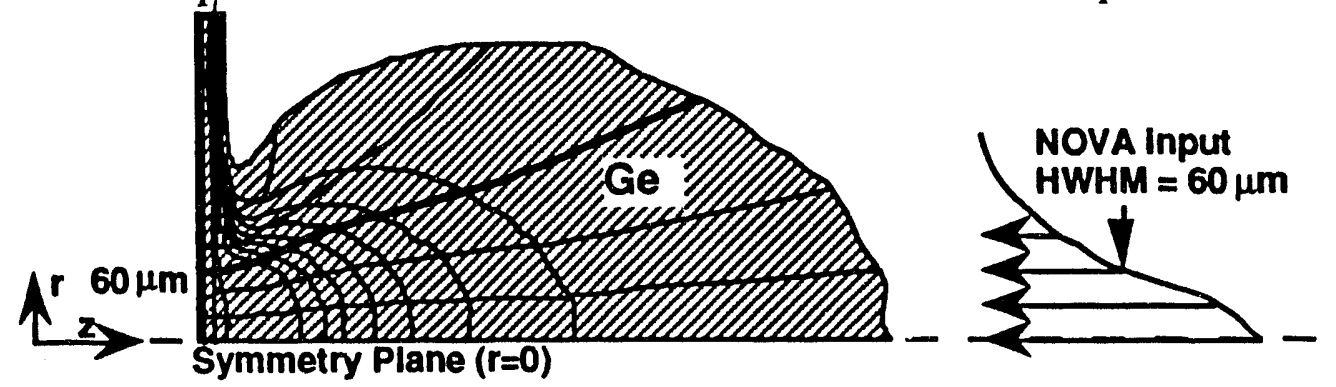

Figure 2. LASNEX-calculated 2-D plasma expansion due to 120- $\mu \mathrm{m}$ (FWHM) line-focused Nova laser incident on uniformly coated Ge slab target, 500 ps into the pulse.

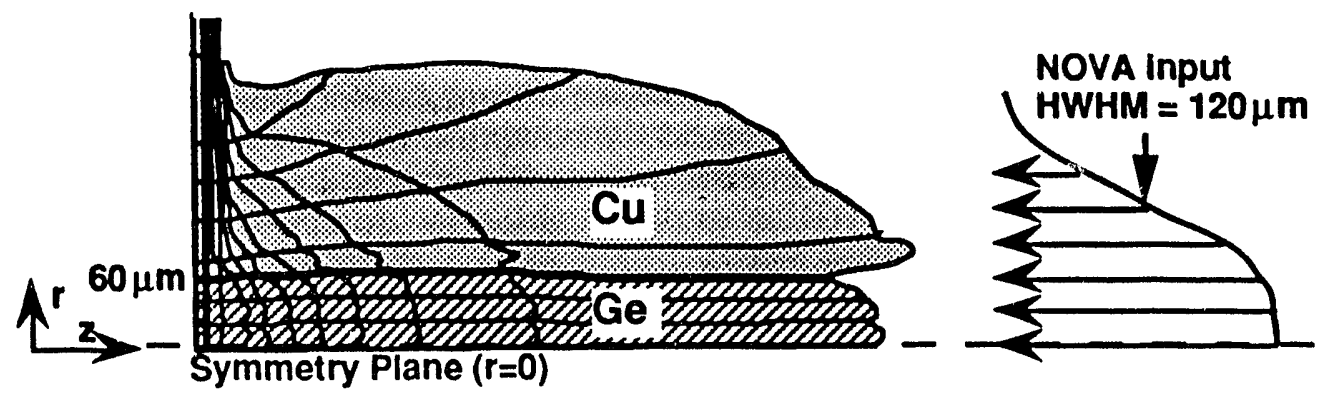

Figure 3. 2-D plasma expansion due to $240-\mu \mathrm{m}$ line-focused Nova laser incident on $120-\mu \mathrm{m}$ Ge stripe coated on solid $\mathrm{Cu}$ substrate, $500 \mathrm{ps}$ into the pulse. 


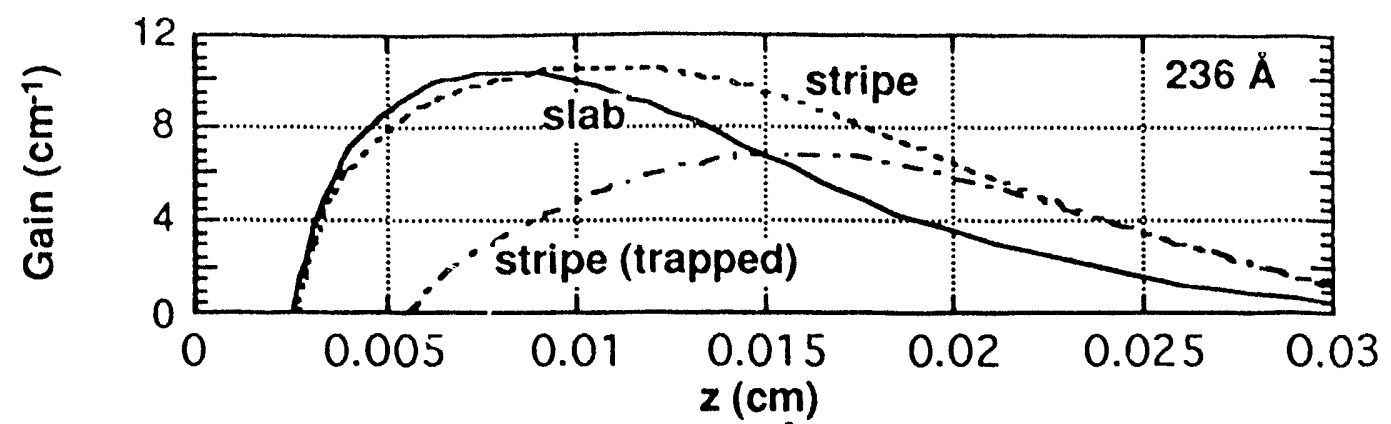

Figure 4. Spatial gain profiles of the Ge $236-\AA$ line, at the $r=0$ symmetry plane, 500 ps into the pulse, between the slab (solid line) and stripe (dotted line) XRLs. The dashdotted line shows the effect of trapping on the gain of the stripe XRL.

excitation, and Auger processes. Rates for inverse processes are done via detailed balance. The accuracy of the gain values is affected by the atomic models and the details of the calculations. Our $\mathrm{Ge}$ atomic model has detailed levels at the Ne-like isoelectronic sequence up to principal quantum number $n=3$. We surround the laser transitions with sufficient detailed atomic structures in order to assure accurate calculations of the level populations.

Figure 4 plots spatial gain profiles of the Ge $236-\AA$ line, along the $\mathrm{r}=0$ symmetry plane, from the simulations shown in Figs. 2 and 3. The peak gain values are similar, but we observe a slightly larger gain region for the stripe XRL plasma. The populations are also affected by plasma opacities, such as the effect of radiation trapping. The dash dotted line of Fig. 4 shows the effect of trapping, calculated by formal line transfer, on gain of the $236-\AA$ line for the stripe simulation. We transferred all El lines under the assumption of complete redistribution with account of the effect of Doppler and followed the accuracy guideline prescribed by Eder.(12) With trapping the peak gain of the $236-\AA$ line reduces from $10 \mathrm{~cm}^{-1}$ to $7 \mathrm{~cm}^{-1}$, approaching the measured gain value of $4 \mathrm{~cm}^{-1}$ (13) In an expanding plasma, the density reduces monotonically away from the target surface. With the density reduction the effect of trapping also reduces as shown by the comparisons between the trapped and untrapped cases.

In our 1-D line transfer calculations we assume that the other dimensions are optically thick. We are developing a new 2-D line transfer package for GLF which allows for more realistic simulations. With 2-D spatial gain and $\mathrm{n}_{\mathrm{e}}$ profiles, we can perform ray propagation calculations to match the simulations with actual observable quantities such as laser output intensities. For strongly refractive plasma medium where the refractive length is shorter than the saturation length, we will observe smaller gain values by using the intensity versus laser length ratio method. The gain vaike shown in Fig. 4 would need to be further modified by the effect of refraction. We are currently working on a three dimensional Monte-Carlo ray propagation code to study the effect of refraction and gain saturation,

From the results of the numerical simulations we do not expect the stripe and slab XRLs to perform very differently. However, the best way to find out how well our shaped XRLs work is to experimentally compare the performance of stripe and bowtie XRLs with the conventional line-focused slab XRLs and to perform detailed experimental characterization of shaped XRLs in the laboratory.

\section{EXPERIMENTAL SETUP AND RESULTS}

In our first set of experiments we contrasted the properties of stripe and bowtie XRLs with $120 \mu \mathrm{m}$ best-focused beam illuminating solid Ge slab targets. The stripe XRL is an $120-\mu \mathrm{m}$ wide, $0.2-\mu \mathrm{m}$-thick $\mathrm{Ge}$ stripe deposited on a thick $(3 \mathrm{~mm}) \mathrm{Cu}$ substrate. The bowtie XRL has 
a width of $120 \mu \mathrm{m}$ at the ends and $25 \mu \mathrm{m}$ at the neck of the bowtie. We overfill the stripe and bowtie XRLs by defocusing the incoming Nova beam to $240 \mu \mathrm{m}$ (FWHM) line width. We used a $6(0) \mathrm{ps}$ square pulse at $2(1)$ with an intensity of $20 \mathrm{TW} / \mathrm{cm}^{2}$ to illuminate stripe, bowtie, and slab targets. Diagnostics used for the ongoing characterization of the stripe XRL includes on- and off-axis spectrometers, a pin-hole camera, and a coherence camera. ${ }^{(14)}$

Keanetech is an off-axis streaked $x$-ray crystal spectrograph. We measured the emission spectrum from $8-11 \AA$ which includes most of the Ne-like Ge El lines. The measured stripe spectrum also shows large $\mathrm{Cu}$ emission mixed with $\mathrm{Ge}$ lines. The $\mathrm{Cu}$ emission includes contributions ranging from $\mathrm{N}$ - to $\mathrm{Ne}$-likes and is predominantly in the 9.8 $11 \AA$ range. With more $\mathrm{Cu}$ exposed to the Nova beams, the stripe target has stronger $\mathrm{Cu}$ contributions and weaker Ge contributions than the slab target. However, time histories of the Ge $3 \mathrm{~d}-2 \mathrm{p}$ line emissions between stripe and slab XRL plasmas show similar temporal characteristics, indicating similar ionization histories. The predicted Ne-like populations from our numerical simulations show large Ne-like populations throughout the 600 ps pulse, which also matches the measured emission profiles.

Looking from $45^{\circ}$ down into the expanding plasma and with no spectral resolving power, the GAX pin-hole camera results can provide a qualitative view of plasma expansion. We preferentially filters the longer wavelength emission of $\mathrm{Cu}$ near $12 \AA$. We observed a double-hump spatial emission profile from the overfilled stripe configuration while the bestline-focused slab configuration showed a peaked single-hump emission profile. The doublehump feature of the stripe XRL suggests strong $\mathrm{Cu}$ emission flanking the weaker $\mathrm{Ge}$ emission at the center. We are currently planning a series of experiments to characterize the plasma expansion and interface mixing of a Ge-stripe tamped by different- $\mathrm{Z}$ materials.

A st. aked flat-field spectrograph provides temporally and spectrally resolved data with integrated angular coverage in the blow-off direction (normal to the target). Measurements of XRL lasing lines for 2.9-cm-long stripe XRLs show factors of 3 - 10 weaker intensities as compared to the comparable-length slab XRLs. However, at 3.75-cmlength, the stripe intensity is about the same as for the slab. The weaker $2.9-\mathrm{cm}$ stripe could be due to a number of reasons, including refraction and gain variations. Below saturation minor changes in gain could result in large laser output variations. The $3.75-\mathrm{cm}-$ long bowtie intensity for the $236-\AA$ line is about a factor of 15 weaker than the similar-length stripe and slab XRLs. At this length and assuming a gain of. $4 \mathrm{~cm}^{-1}$ (13) we expect the XRL to be nearly saturated. The comparable output intensities of the $3.75-\mathrm{m}$-long stripe and slab XRLs indicate that at or near saturation, minor differences in gain and ray propagation become negligible, confirming the small discrepancies we found in our numerical simulations. The weaker bowtie XRL can be attributed to factors such as smaller gain medium, mixing at the $\mathrm{XRL} /$ substrate plasma interfaces, and refraction.

A microchannel plate-intensified grazing-incident spectrograph (MCPIGS) measures the time-integrated, spectrally resolved laser footprint. For solid and foil targets we typically measured a wide 15 mrad footprint in the blow-off direction with peak intensity at $\sim 15$ mrad off-axis. When we rotated MCPIGS by $90^{\circ}$ and position the slit at $15 \mathrm{mrad}$ off-axis, we observe a wide angular footprint that is relatively flat over the 10 -mrad angular coverage. To reduce the effect of refraction we need to reduce the $n_{e}$ gradient. By using a prepulse to set up a lower density plasma for the main laser pulse, lasing occurs at flatter density regime which allows for significant reduction on the effect of refraction. Detailed descriptions on the effect of prepulse can be found in the work of Nilsen. ${ }^{(15)}$ We have fielded one Ni-stripe XRL (coated on $\mathrm{Cu}$ ) with prepulse and found that the output laser intensity in the blow off direction peaks at 5 mrad off-axis, significantly lower than previously measured output without prepulse. Another characteristic of the prepulsed XRLs is strong $J=0-1$ laser line 
dominating the entire laser spectrum. The strong $\mathrm{J}=()-1 \mathrm{Ni}$-line has wavelength of $231 \AA$, and in a regime where we have abundance of multilayer mirrors for coherence diagnostics.

\section{SUMMARY}

A bowtie-shaped $x$-ray laser has been proposed as a spatially adaptive filter technique to achieve saturated output with diffraction-limited transverse coherence. We have performed numerical simulations and a series of Nova experiments to characterize a simpler stripe XRL as the first step in demonstrating our ability to control the XRL shape during plasma expansion. From 2-D LASNEX calculations we found that similar-Z hydrodynamic tamper, such as $\mathrm{Cu}$ on Ge XRL, can effectively control the XRL plasma expansion to 1-D. Pin-hole camera images contrasting stripe and slab emission features qualitatively confirmed our LASNEX results. We also measured comparable temporal histories of identified Ne-like Ge $3 d-2 p$ emission lines between the slab and stripe XRLs which indicate comparable ionization histories. At or near saturation the stripe XRL intensities are comparable to a linefocused slab XRL, indicating no significant differences in gains and refractive ray propagation between the two laser configurations. The bowtie XRL intensities were factors of $\sim 15$ lower than the nearly-saturated, similar-length stripe and slab XRLs. This intensity difference could be due to refraction, mixing, or the geometric differences. However, the coherent power of a saturated bowtie should be stronger than other XRL configurations.

We are planning further characterization of stripe and bowtie XRLs, such as measurements of small signal gains, plasma expansion characteristics, and coherence. Preliminary prepulse experiments on a Ni XRL show the promise of a low-divergence, strong output-intensity XRL for further coherence study. We also have plans to further enhance the bowtie output by varying Nova pulse shapes, using new XRL target designs, and using multiple-staged oscillator-amplifier configurations.

\section{REFERENCES}

1. D. Matthews et al., Phys. Rev. Lett. 54, 110-113 (1985).

2. Broceedings of the Applications of X-ray Lasers Workshop, R. A. London, D. L. Matthews, S. Suckewer, editors, LLNL Report CONF-9206170 (January 1992).

3. J. A. Koch et al., Phys. Rev. Lett. 68, 3291-3294 (1992).

4. P. Jaegle et al, X-Ray Laser 1992, E. Fill, editor, 1-7 (IOP Publishing, Bristol and Philadelphia, 1992).

5. J. E. Trebes et al., Phys. Rev. Lett. 68, 588-591 (1992).

6. C. L. S. Lewis et al., Opt. Commun. 91, 71-76 (1992).

7. B. J. MacGowan et al., L of X-ray Science and Tech, 3, 231-282 (1993).

8. S. B. Libby, R. A, London, and T. Weaver, "New configurations for high coherent power X-ray lasers," to be published.

9. A. S. Wan et al., X-Ray Laser 1992, E. Fill, editor, 293-296 (IOP Publishing, Bristol and Philadelphia, 1992).

10. G. B. Zimmerman, W. L. Kruer, Com. Plasma Phys, and Cont. Fusion 2, 51-61 (1975).

11. H. A. Scott, R. W. Mayle, LLNL Report No. UCRL JC-114510 (June 1993), to be published in Appl. Phys. B.

12. D. C. Eder, H. A. Scoth, L. Quant. Sposc. Radiat. Transfer. 45, 189 (1991)

13. C. L S. Lewis et al., X-Ray Laser 1990, G. J. Tallents, editor, 231-238 (IOP Publishing, Bristol and Philadelphia, 1991).

14. A. S. Wan et al., LLNL Report No. UCRL JC-112553 (July 1993), to be published in Optical Engineering.

15. J. Nilsen et al., LLNL, Report No. UCRL JC-112704 (January 1993). 

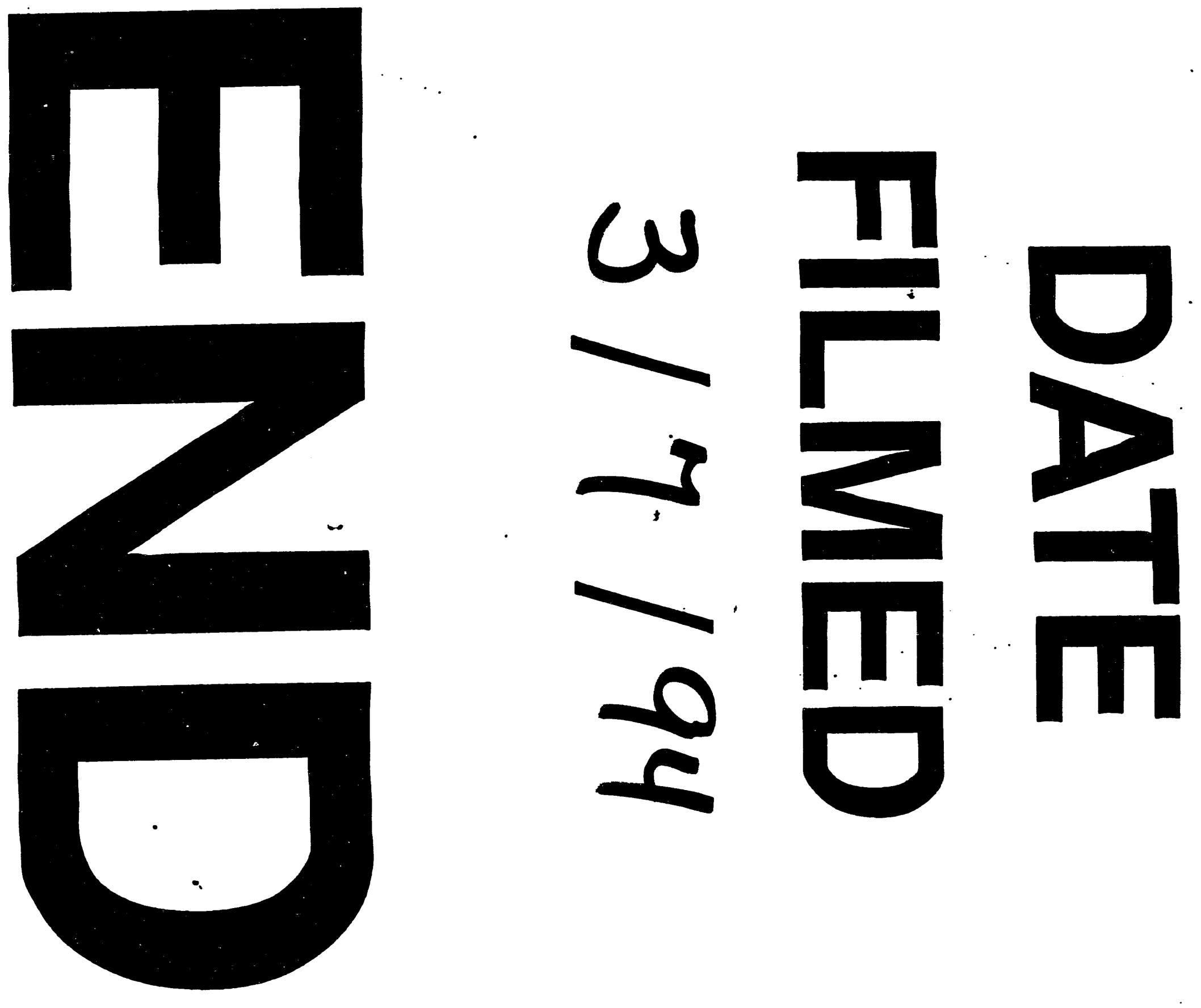
